\title{
Diphoton Signatures from Heavy Axion Decays at the CERN Large Hadron Collider
}

DOI:

10.1103/PhysRevD.93.015017

\section{Document Version}

Final published version

Link to publication record in Manchester Research Explorer

\section{Citation for published version (APA):}

Pilaftsis, A. (2016). Diphoton Signatures from Heavy Axion Decays at the CERN Large Hadron Collider. Physical Review D (Particles, Fields, Gravitation and Cosmology), D93, 1-3. [ 015017].

https://doi.org/10.1103/PhysRevD.93.015017

\section{Published in:}

Physical Review D (Particles, Fields, Gravitation and Cosmology)

\section{Citing this paper}

Please note that where the full-text provided on Manchester Research Explorer is the Author Accepted Manuscript or Proof version this may differ from the final Published version. If citing, it is advised that you check and use the publisher's definitive version.

\section{General rights}

Copyright and moral rights for the publications made accessible in the Research Explorer are retained by the authors and/or other copyright owners and it is a condition of accessing publications that users recognise and abide by the legal requirements associated with these rights.

\section{Takedown policy}

If you believe that this document breaches copyright please refer to the University of Manchester's Takedown Procedures [http://man.ac.uk/04Y6Bo] or contact uml.scholarlycommunications@manchester.ac.uk providing relevant details, so we can investigate your claim.

\section{OPEN ACCESS}


PHYSICAL REVIEW D 93, 015017 (2016)

\title{
Diphoton signatures from heavy axion decays at the CERN Large Hadron Collider
}

\author{
Apostolos Pilaftsis \\ Consortium for Fundamental Physics, School of Physics and Astronomy, University of Manchester, \\ Manchester M13 9PL, United Kingdom
}

(Received 16 December 2015; published 27 January 2016)

\begin{abstract}
Recently, the LHC collaborations, ATLAS and CMS, have announced an excess in the diphoton channel with local significance of about $3 \sigma$ around an invariant mass distribution of $\sim 750 \mathrm{GeV}$, after analyzing new data collected at center-of-mass energies of $\sqrt{s}=13 \mathrm{TeV}$. We present a possible physical interpretation of such a signature, within the framework of a minimal UV-complete model with a massive singlet pseudoscalar state $a$ that couples to a new TeV-scale colored vectorlike fermion $F$, whose hypercharge quantum number is a non-zero integer. The pseudo-scalar state $a$ might be a heavy pseudo-Goldstone boson, such as a heavy axion, which decays into two photons and whose mass lies around the excess region. The mass of the $C P$-odd state $a$ and its coupling to $F$ may be due to nonperturbative effects, which can break the original Goldstone shift symmetry dynamically. The possible role that the heavy axion $a$ can play in the radiative generation of a seesaw Majorana scale and in the solution to the so-called strong $C P$ problem is briefly discussed.
\end{abstract}

DOI: $10.1103 /$ PhysRevD.93.015017

Recently, the ATLAS and CMS collaborations have analyzed Run 2 LHC data gathered at center-of-mass energies of $\sqrt{s}=13 \mathrm{TeV}$. They reported an excess in the diphoton channel around an invariant mass distribution of $\sim 750 \mathrm{GeV}$, with local significance of $3.6 \sigma$ and $2.6 \sigma$ confidence level (CL), respectively [1]. Interestingly enough, Run 2 data do not show up any significant excess in other diboson channels, such as $Z Z, W^{+} W^{-}$and $Z \gamma$, whilst the Run-1 bumps seen around the $2 \mathrm{TeV}$ region have now become almost statistically insignificant.

In this short paper, we offer a possible interpretation of such an excess in the diphoton channel, within the framework of a minimal UV-complete model with a massive singlet pseudoscalar state $a$ that couples to new colored vectorlike fermions $F_{L, R}$. These vectorlike fermions are very heavy with masses $m_{F} \gtrsim 1.5 \mathrm{TeV}$, so as to have escaped detection so far at the LHC. They are charged under the $\mathrm{SU}(3)_{C}$ group of quantum chromodynamics (QCD), but they are singlets under the weak $\mathrm{SU}(2)_{L}$ group of the standard model (SM). They must also have nonzero integer hypercharges, e.g., $Y_{F_{L}}=Y_{F_{R}}=1,2, \ldots$, which forbid them to have Yukawa interactions with the SM quarks. On the other hand, the pseudoscalar state $a$ may well be a heavy pseudo-Goldstone boson, such as a heavy axion, which decays into two photons with a mass that lies around the excess region of $\sim 750 \mathrm{GeV}$. Both the mass $M_{a}$ of the state $a$ and its $C P$-odd coupling to the Dirac vectorlike fermion $F, h_{F} \bar{F} i \gamma_{5} F$, could originate from nonperturbative effects that break the original axion shift symmetry.

The minimal UV-complete model that we will be considering here is related to the one that discussed earlier in Ref. [2]. The relevant non-SM part of the Lagrangian of interest to us is given by
$\mathcal{L}=\bar{F}\left(i D-m_{F}\right) F+\frac{1}{2}\left(\partial_{\mu} a\right)\left(\partial^{\mu} a\right)-\frac{1}{2} M_{a}^{2} a^{2}-h_{F} a \bar{F} i \gamma_{5} F$.

In the above, $D_{\mu}=\partial_{\mu}+i g_{s} T^{a} G_{\mu}^{a}+i g^{\prime}\left(Y_{F} / 2\right) B_{\mu}$ is the covariant derivative acting on the exotic colored Dirac fermion $F$, where $G_{\mu}^{a}$ and $B_{\mu}$ are the $\mathrm{SU}(3)_{C}$ and $\mathrm{U}(1)_{Y}$ gauge bosons, respectively, and $T^{a}$ (with $a=1,2, \ldots, 8$ ) are the generators of the $\mathrm{SU}(3)_{C}$ gauge group. Notice that Lagrangian (1) is invariant under the $C P$ transformations: $a(t, \mathbf{x}) \rightarrow-a(t,-\mathbf{x})$ and $\bar{F}(t, \mathbf{x}) i \gamma_{5} F(t, \mathbf{x}) \rightarrow-\bar{F}(t,-\mathbf{x})$ $i \gamma_{5} F(t,-\mathbf{x})$. In the absence of the fermion mass term $m_{F} \bar{F} F$, Lagrangian (1) is also invariant under the chirality discrete transformations: $a \rightarrow-a$ and $F_{R(L)} \rightarrow+(-) F_{R(L)}$. Given that $m_{F} \neq 0$, this latter symmetry is broken softly by the dimension-3 mass operator $m_{F} \bar{F} F$. Finally, it is important to remark that the squared mass $M_{a}^{2}$ and the Yukawa couplings $h_{F}$ in Lagrangian (1) break explicitly the Goldstone shift symmetry: $a \rightarrow a+c$, where $c$ is an arbitrary constant. The possible origin of such a breaking could be due to nonperturbative effects related to some unspecified strong dynamics.

In the above minimal extension of the SM, the pseudoscalar field $a$ couples to the electromagnetic (em) field $A_{\mu}$ and the gluon fields $G_{\mu}^{a}$, via the five-dimensional operators: $a F^{\mu \nu} \tilde{F}_{\mu \nu}$ and $a G^{a \mu \nu} \tilde{G}_{\mu \nu}^{a}$, where $F^{\mu \nu}$ and $G^{a, \mu \nu}$ are the $\mathrm{U}(1)_{\mathrm{em}}$ and $\mathrm{SU}(3)_{C}$ field strength tensors, respectively, and $\tilde{F}_{\mu \nu} \equiv$ $\frac{1}{2} \varepsilon_{\mu \nu \lambda \rho} F^{\lambda \rho}$ and $\tilde{G}_{\mu \nu}^{a} \equiv \frac{1}{2} \varepsilon_{\mu \nu \lambda \rho} G^{a, \lambda \rho}$ are their corresponding dual tensors. These operators are induced by the chiral global anomalies of the heavy fermion $F$, through the triangle graphs shown in Fig. 1. With the convention that all 


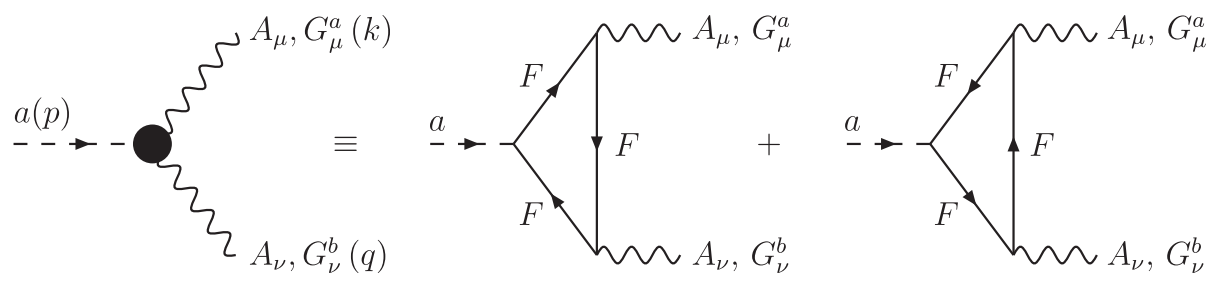

FIG. 1. The operators $a F_{\mu \nu} \tilde{F}^{\mu \nu}$ and $a G_{\mu \nu}^{a} \tilde{G}_{\mu \nu}^{a}$, as induced by the chiral global anomaly of the heavy fermion $F$, with the convention $p+k+q=0$.

momenta are incoming, i.e., $p+k+q=0$, the one-loop $a(p)-A_{\mu}(k)-A_{\nu}(q)$ coupling reads [3-5]:

$i \Gamma_{\mu \nu}^{a A A}(p, k, q)=i Q_{F}^{2} \frac{N_{C} \alpha_{\mathrm{em}}}{\pi} \frac{h_{F}}{m_{F}} F_{P}\left(\frac{p^{2}}{4 m_{F}^{2}}\right) \varepsilon_{\mu \nu \lambda \rho} k^{\lambda} q^{\rho}$,

where $Q_{F}=Y_{F} / 2$ is the electric charge of the heavy fermion $F, N_{C}=3$ is its color degrees of freedom, $\alpha_{\mathrm{em}}=$ $e^{2} /(4 \pi)$ is the electromagnetic fine structure constant, and $\varepsilon_{\mu \nu \lambda \rho}$ is the usual antisymmetric Levi-Civita tensor (with the convention: $\varepsilon^{0123}=+1$ ). Moreover, the loop function $F_{P}(\tau)$ was calculated a long time ago [3] and found to be

$$
F_{P}(\tau)= \begin{cases}\frac{1}{\tau} \arcsin ^{2} \sqrt{\tau} ; & |\tau| \leq 1, \\ -\frac{1}{4 \tau}\left[\ln \left(\frac{\sqrt{\tau}+\sqrt{\tau-1}}{\sqrt{\tau}-\sqrt{\tau-1}}\right)-i \pi\right]^{2} ; & |\tau| \geq 1 .\end{cases}
$$

Note that for $|\tau| \ll 1$, we have $F_{P}(\tau)=1+\tau / 3+\mathcal{O}\left(\tau^{2}\right)$, whereas for $|\tau| \gg 1, F_{P}(\tau) \rightarrow-\ln ^{2}|\tau| /(4 \tau)$ which goes to zero asymptotically as $\tau \rightarrow \infty$.

By analogy, the $\mathrm{SU}(3)_{C}$ global anomaly generates an effective interaction of the heavy axion $a$ to gluons $G_{\mu}^{a}$, as shown in Fig. 1. The effective $a(p)-G_{\mu}^{a}(k)-G_{\nu}^{b}(q)$ coupling is given by

$$
i \Gamma_{\mu \nu}^{a G^{a} G^{b}}(p, k, q)=i \delta^{a b} \frac{\alpha_{s}}{2 \pi} \frac{h_{F}}{m_{F}} F_{P}\left(\frac{p^{2}}{4 m_{F}^{2}}\right) \varepsilon_{\mu \nu \lambda \rho} k^{\lambda} q^{\rho},
$$

where $\alpha_{s}=g_{s}^{2} /(4 \pi)$ is the strong fine structure constant.

With the aid of the effective couplings given in (2) and (4), it is straightforward to calculate the decay widths of the heavy axion $a$ into photons $(\gamma)$ and gluons $(g)$ :

$$
\begin{gathered}
\Gamma(a \rightarrow \gamma \gamma)=\frac{N_{C}^{2} \alpha_{\mathrm{em}}^{2}}{64 \pi^{3}} Q_{F}^{4} h_{F}^{2} \frac{M_{a}^{3}}{m_{F}^{2}}\left|F_{P}\left(\tau_{a}\right)\right|^{2}, \\
\Gamma(a \rightarrow g g)=\frac{\alpha_{s}^{2}}{32 \pi^{3}} h_{F}^{2} \frac{M_{a}^{3}}{m_{F}^{2}}\left|F_{P}\left(\tau_{a}\right)\right|^{2} K_{a}^{g},
\end{gathered}
$$

where $\tau_{a} \equiv M_{a}^{2} /\left(4 m_{F}^{2}\right)$ and $K_{a}^{g} \approx 1.6$ is a QCD loop enhancement factor which includes the leading order QCD corrections [6]. In addition, the other diboson decay channels, such as $a \rightarrow Z Z, Z \gamma$ and $W^{+} W^{-}$, may be reliably estimated to leading order in $M_{Z}^{2} / M_{a}^{2}$ [7] as follows:

$$
\begin{aligned}
& \frac{\Gamma(a \rightarrow Z Z)}{\Gamma(a \rightarrow \gamma \gamma)} \approx \frac{\sin ^{4} \theta_{w}}{\cos ^{4} \theta_{w}} \approx 0.082, \\
& \frac{\Gamma(a \rightarrow Z \gamma)}{\Gamma(a \rightarrow \gamma \gamma)} \approx \frac{2 \sin ^{2} \theta_{w}}{\cos ^{2} \theta_{w}} \approx 0.57,
\end{aligned}
$$

whilst the decay width $a \rightarrow W^{+} W^{-}$is negligible, since the corresponding $a W^{+} W^{-}$effective coupling is generated at the two-loop level, e.g., from the one-loop induced $a \gamma \gamma$ coupling. To satisfy the LHC constraints on the masses of exotic colored fermions, we may assume that the vectorlike fermion $F$ is heavier than $a$, e.g., $m_{F} \gtrsim 1.5 \mathrm{TeV}$, in which case $\tau_{a} \ll 1$. Hence, the loop function $F_{P}\left(\tau_{a}\right)$ may well be approximated as $F_{P}\left(\tau_{a}\right) \approx 1$.

If we now take the ratio $R$ of the photonic versus the gluonic decay width given in (5) and (6), we readily find that

$$
R \equiv \frac{\Gamma(a \rightarrow \gamma \gamma)}{\Gamma(a \rightarrow g g)}=\frac{N_{C}^{2} \alpha_{\mathrm{em}}^{2} Q_{F}^{4}}{2 \alpha_{s}^{2} K_{a}^{g}} .
$$

Observe that the ratio $R$ is independent of the Yukawa coupling $h_{F}$ and, for $Q_{F} \geq 2$, we obtain $R>1$ and the decay $a \rightarrow \gamma \gamma$ can easily become the dominant mode.

The production cross section of heavy axions via gluongluon fusion [8] may be calculated as follows:

$$
\sigma(p p \rightarrow a \rightarrow \gamma \gamma) \approx \sigma(p p \rightarrow a) B(a \rightarrow \gamma \gamma),
$$

where $B(a \rightarrow \gamma \gamma) \approx R /(1+1.57 R)$ is the branching fraction for the decay channel $a \rightarrow \gamma \gamma$, with $R$ given in (8). For center-of-mass energies of $\sqrt{s}=13 \mathrm{TeV}$, we may naively estimate the cross section $\sigma(p p \rightarrow a)$ as

$$
\sigma(p p \rightarrow a) \sim \sigma_{\mathrm{SM}}(p p \rightarrow H) \times h_{F}^{2} \frac{m_{t}^{2}}{m_{F}^{2}} \frac{M_{a}^{2}}{M_{H}^{2}},
$$

where $\sigma_{\mathrm{SM}}(p p \rightarrow H) \approx 40 \mathrm{pb}$ is a reference production cross section of the SM Higgs boson $H$ via gluon-gluon fusion, with $M_{H} \approx 125 \mathrm{GeV}$ [9]. Hence, for $M_{a}=$ $750 \mathrm{GeV}$ (or $M_{a} / M_{H}=6$ ), $m_{F} / m_{t}=10$ and $h_{F}=0.1$, we find that

$$
\sigma(p p \rightarrow a \rightarrow \gamma \gamma) \sim 15 \mathrm{fb} \times B(a \rightarrow \gamma \gamma) .
$$


For $B(a \rightarrow \gamma \gamma) \sim 1$ and an integrated luminosity $\mathcal{L}=$ $3 \mathrm{fb}^{-1}$ at $\sqrt{s}=13 \mathrm{TeV}$, we obtain about 45 signal events, which is compatible with the diphoton-excess events reported in [1].

As discussed in detail in [2], axionlike fields could act as mediators to generate $\mathrm{TeV}$-scale gauge-invariant masses, such as $m_{F} \bar{F} F$, for vectorlike fermions through global anomalies at the three-loop level. In particular, a gaugeinvariant Majorana mass term $m_{M}\left(\bar{\nu}_{R}\right)^{C} \nu_{R}$ can be generated [10], if heavy axion fields couple to Kalb-Ramond axions $[11,12]$ that occur in torsionful theories of quantum gravity. Light axions play an important role in solving the strong $C P$ problem via the so-called Peccei-Quinn mechanism [13-16]. Thus, the possible presence of a heavy axion, or a multitude of axions [17,18], may give rise to interesting mixing phenomena and possibly to new effects in astrophysical considerations [19].

In conclusion, we have presented a minimal UVcomplete model, based on the possible existence of a heavy axion with mass $M_{a} \approx 750 \mathrm{GeV}$, which could offer a possible physical interpretation of the diphoton excess observed in the Run 2 data. The model requires the presence of a new $\mathrm{TeV}$-scale colored vectorlike fermion $F$, which has a nonzero integer hypercharge. For large electric charges $Q_{F} \geq 2$, the photonic decay mode $a \rightarrow \gamma \gamma$ becomes naturally the dominant channel. The latter, along with the branching fractions given in (7), provide a unique prediction of our minimal model that can be tested with future Run 2 data. Nevertheless, our model may require an extension to its field content, as it exhibits a Landau pole at energy scales $Q \lesssim 10^{14} \mathrm{GeV}$, for $Q_{F} \geq 2$. Further studies are therefore needed, so as to be able to fully assess the physical significance of the observed diphoton excess, as a firm signature of new physics at the LHC.

\section{ACKNOWLEDGMENTS}

The author thanks Andrew Pilkington for discussions on the ATLAS signal events. This work is supported in part by the Lancaster-Manchester-Sheffield Consortium for Fundamental Physics, under STFC Research Grant No. ST/L000520/1.
[1] Public presentations on ATLAS and CMS physics results from Run 2 (on 15/12/2015): CMS results (Speaker: Jim Olsen), based on the CMS Report No. CMS PAS EXO-15004; ATLAS results (Speaker: Marumi Kado), based on the ATLAS Report No. ATLAS-CONF-2015-081.

[2] A. Pilaftsis, Anomalous fermion mass generation at three loops, Mod. Phys. Lett. A 28, 1350083 (2013).

[3] J. Steinberger, On the use of subtraction fields and the lifetimes of some types of meson decay, Phys. Rev. 76, 1180 (1949).

[4] S. L. Adler, Axial vector vertex in spinor electrodynamics, Phys. Rev. 177, 2426 (1969).

[5] J. S. Bell and R. Jackiw, A PCAC puzzle: $\pi^{0} \rightarrow \gamma \gamma$ in the sigma model, Nuovo Cimento A 60, 47 (1969).

[6] M. Spira, A. Djouadi, D. Graudenz, and P. M. Zerwas, Higgs boson production at the LHC, Nucl. Phys. B453, 17 (1995).

[7] R. Brustein and D. H. Oaknin, Signatures of hypercharge axions in colliders, Phys. Rev. D 62, 015001 (2000).

[8] J. Jaeckel, M. Jankowiak, and M. Spannowsky, LHC probes the hidden sector, Phys. Dark Univ. 2, 111 (2013).

[9] K. A. Olive (Particle Data Group Collaboration), Review of particle physics, Chin. Phys. C 38, 090001 (2014).

[10] N. E. Mavromatos and A. Pilaftsis, Anomalous Majorana neutrino masses from torsionful quantum gravity, Phys. Rev. D 86, 124038 (2012).
[11] M. Kalb and P. Ramond, Classical direct interstring action, Phys. Rev. D 9, 2273 (1974).

[12] M. J. Bowick, S. B. Giddings, J. A. Harvey, G. T. Horowitz, and A. Strominger, Axionic Black Holes and a BohmAharonov Effect for Strings, Phys. Rev. Lett. 61, 2823 (1988).

[13] R. D. Peccei and H. R. Quinn, $C P$ Conservation in the Presence of Instantons, Phys. Rev. Lett. 38, 1440 (1977).

[14] R. D. Peccei and H. R. Quinn, Constraints imposed by $C P$ conservation in the presence of instantons, Phys. Rev. D 16, 1791 (1977).

[15] S. Weinberg, A New Light Boson?, Phys. Rev. Lett. 40, 223 (1978).

[16] F. Wilczek, Problem of Strong $P$ and $T$ Invariance in the Presence of Instantons, Phys. Rev. Lett. 40, 279 (1978).

[17] A. Arvanitaki, S. Dimopoulos, S. Dubovsky, N. Kaloper, and J. March-Russell, String axiverse, Phys. Rev. D 81, 123530 (2010).

[18] M. Cicoli, M. Goodsell, and A. Ringwald, The type IIB string axiverse and its low-energy phenomenology, J. High Energy Phys. 10 (2012) 146.

[19] P. Tinyakov, I. Tkachev, and K. Zioutas, Tidal streams from axion miniclusters and direct axion searches, arXiv:1512.02884. 